\title{
Structural vs electronic origin of renormalized band widths in TTF-TCNQ: An angular dependent NEXAFS study
}

Sing, M; Meyer, J; Hoinkis, M; Glawion, S; Blaha, P; Gavrila, G; Jacobsen, Claus Schelde; Claessen, R

Published in:

Physical Review B Condensed Matter

Link to article, DOI:

10.1103/PhysRevB.76.245119

Publication date:

2007

Document Version

Publisher's PDF, also known as Version of record

Link back to DTU Orbit

Citation (APA):

Sing, M., Meyer, J., Hoinkis, M., Glawion, S., Blaha, P., Gavrila, G., Jacobsen, C. S., \& Claessen, R. (2007).

Structural vs electronic origin of renormalized band widths in TTF-TCNQ: An angular dependent NEXAFS study.

Physical Review B Condensed Matter, 76(24), 245119. https://doi.org/10.1103/PhysRevB.76.245119

\section{General rights}

Copyright and moral rights for the publications made accessible in the public portal are retained by the authors and/or other copyright owners and it is a condition of accessing publications that users recognise and abide by the legal requirements associated with these rights.

- Users may download and print one copy of any publication from the public portal for the purpose of private study or research.

- You may not further distribute the material or use it for any profit-making activity or commercial gain

- You may freely distribute the URL identifying the publication in the public portal 


\title{
Structural vs electronic origin of renormalized band widths in TTF-TCNQ: An angular dependent NEXAFS study
}

\author{
M. Sing, ${ }^{1, *}$ J. Meyer, ${ }^{1}$ M. Hoinkis,,${ }^{1,2}$ S. Glawion, ${ }^{1}$ P. Blaha, ${ }^{3}$ G. Gavrila,${ }^{4}$ C. S. Jacobsen,${ }^{5}$ and R. Claessen ${ }^{1}$ \\ ${ }^{1}$ Experimentelle Physik IV, Universität Würzburg, D-97074 Würzburg, Germany \\ ${ }^{2}$ Experimentalphysik II, Universität Augsburg, D-86135 Augsburg, Germany \\ ${ }^{3}$ Institute of Materials Chemistry, Vienna University of Technology, A-1060 Vienna, Austria \\ ${ }^{4}$ Institut für Physik, Technische Universität Chemnitz, D-09107 Chemnitz, Germany \\ ${ }^{5}$ Department of Physics, Technical University of Denmark, DK-2800 Lyngby, Denmark
}

(Received 22 August 2007; published 20 December 2007)

\begin{abstract}
We have performed angle-dependent near-edge x-ray absorption fine structure measurements in the Auger electron yield mode on the correlated quasi-one-dimensional organic conductor tetrathiafulvalenetetracyanoquinodimethane (TTF-TCNQ) in order to determine the orientation of the molecules in the topmost surface layer. We find that the tilt angles of the molecules with respect to the one-dimensional axis are essentially the same as in the bulk. Thus, we can rule out surface relaxation as the origin of the renormalized band widths which were inferred from the analysis of photoemission data within the one-dimensional Hubbard model. Thereby, recent theoretical results are corroborated which invoke long-range Coulomb repulsion as alternative explanation to understand the spectral dispersions of TTF-TCNQ quantitatively within an extended Hubbard model.
\end{abstract}

DOI: $10.1103 /$ PhysRevB.76.245119

PACS number(s): 71.20.Rv, 61.10.Ht, 71.10.Pm

\section{INTRODUCTION}

One-dimensional (1D) electron systems represent a valuable test case for the understanding of unusual quantum many-body states. On theoretical grounds, the enhanced correlations in one dimension lead to a breakdown of the paradigmatic Fermi liquid picture involving new generic lowenergy excitations. These manifest themselves in a dynamic decoupling of charge and spin degrees of freedom commonly referred to as spin-charge separation. ${ }^{1}$ While the low-energy physics is captured, e.g., within the so-called TomonagaLuttinger model, which assumes linear band dispersions and therefore does not imply any intrinsic energy scale, the simplest model to explore generic 1D physics on the energy scale of the conduction band width is the 1D single-band Hubbard model (HM). ${ }^{2}$ The organic quasi-one-dimensional conductor tetrathiafulvalene-tetracyanoquinodimethane (TTF-TCNQ), where stacks of either the TCNQ or TTF planar molecules are effectively doped and become conducting by charge transfer from TTF to TCNQ, was the first material where angle-resolved photoemission spectroscopy (ARPES) was able to positively identify such 1D single-band HM spectral features as spinon and holon branches and the shadow band. ${ }^{3,4}$ However, in order to achieve a quantitative description of the experimental data, a renormalization of the hopping integral $t$ at the surface by a factor of $\approx 2$ with respect to the bulk value from density-functional theory or estimates from bulk-sensitive measurements had to be assumed. At that time, it was argued that due to the different Madelung potential at the surface, the topmost molecular layer may be relaxed with tilting angles of the molecules against the 1D axis being different from the bulk. An alternative proposition has recently been elaborated on, ${ }^{5}$ namely, the necessity to incorporate the next-nearest-neighbor Coulomb repulsion $V$ in a $\mathrm{HM}$ description in addition to the onsite Coulomb energy $U{ }^{6}$ This reduces the effective energy for two electrons to pass each other to $U-V$ and hence enhances the effective hopping integral $t_{e f f} \approx t U /(U-V)$ without the need to assume a larger $t$. Indeed, it was shown by density-functional theory that $V \approx U / 2$ within a stack. ${ }^{5}$ However, these results are based on the bulk crystal structure, and an experimental proof that no surface reconstruction is at work here is still lacking. There are few experimental techniques which, in principle, could give access to the molecular surface tilt such as quantitative low-energy electron diffraction $I V$ measurements or scanning tunneling microscopy (STM). Nevertheless, these techniques, if applicable at all, provide only indirect information and need theoretical simulations to interpret the experimental results. Here, we employ a method widely used in surface physics to determine orientations of adsorbed molecules and apply it to the surface of TTF-TCNQ, namely, angular dependent near-edge x-ray absorption fine structure (NEXAFS) spectroscopy.?

\section{EXPERIMENTAL AND TECHNICAL DETAILS}

The NEXAFS measurements were performed at the PM-3 beamline of BESSY (Berlin, Germany) using its MUSTANG end station. The energy resolution amounted to $35 \mathrm{meV}$ at the $\mathrm{N} K$ edge and was even lower according to the characteristics of a plane-grating monochromator at the $\mathrm{C} K$ and $\mathrm{S}$ $L$ edges. Clean sample surfaces were exposed through in situ cleavage of TTF-TCNQ single crystals at low temperatures. To reduce radiation damage, all spectra were recorded at $60 \mathrm{~K}$, i.e., above the charge density wave phase transition temperature in the metallic phase. In order to achieve the required surface sensitivity, the absorption signal was monitored in the Auger electron yield mode using a SPECS PHOIBOS 150 analyzer set to kinetic energies of 151, 268, and $381 \mathrm{eV}$ for S-, C-, and N-specific Auger lines, respectively. At these kinetic energies, the electron mean free path is typically less than $10 \AA{ }^{7}$ which compares to the thickness 
of a single molecular layer in TTF-TCNQ of $c / 2=9.23 \AA$, where $c$ is the lattice constant perpendicular to the surface (see below). Hence, the absorption signal indeed stems essentially from the topmost molecular layer. All absorption spectra were corrected for the time and energy dependences of the incoming photon flux through division by the ring current and the drain current of a freshly sputtered Pt foil, respectively. The data were normalized in an energy region about $75 \mathrm{eV}$ above the absorption thresholds, where the final states are basically free-electron-like and hence isotropic. Great care was exercised to avoid sample degradation upon irradiation. The irradiation exposure time of one and the same sample was about $40 \mathrm{~min}$ before significant changes in the photoemission spectra of the S $2 p$ core level were detected which turned out to monitor most sensitively photoninduced damages.

In a molecular orbital picture, NEXAFS involves the resonant x-ray excitation of a $K$-or $L$-shell electron into unoccupied low-lying molecular states governed by dipole selection rules. These project out the $s-, p$-, or $d$-like components of the antibonding $\sigma^{*}$ or $\pi^{*}$ final states at the excited atom, which makes NEXAFS site and element specific. The idea to determine the molecular orientation of molecules on or at surfaces is based on the polarization dependence of the involved dipole matrix elements. Maximum absorption is obtained when the electric field vector $\mathbf{E}$ of the linearly polarized $\mathrm{x}$ rays points along a vectorlike orbital or lies within a planelike orbital. ${ }^{8}$ The absorption intensity then scales as $\cos ^{2} \delta$, where $\delta$ denotes the angle between $\mathbf{E}$ and the direction of a vectorlike orbital or the plane of a planelike orbital. Detailed expressions for the angular dependence of the $K$-shell absorption signal are given in Ref. 9 considering both the polar and azimuthal orientations of molecular orbitals with respect to the sample surface normal and in addition the degree of polarization. We note that in our case, the expressions are simplified and become applicable also for $L$-shell absorption, since the azimuthal orientation of the TTF and TCNQ molecules can be assumed to be fixed and the variation of the $\mathrm{x}$-ray light incidence angle is restricted to the plane spanned by the sample surface normal and the 1D axis. If one, moreover, takes into account that the molecules from adjacent stacks of the same kind are tilted in opposite directions, one finally arrives at the following expressions for the angular dependence of vector- and planelike orbitals, respectively:

$$
\begin{gathered}
I_{v}=C\left(\cos ^{2} \theta \cos ^{2} \alpha+\sin ^{2} \theta \sin ^{2} \alpha\right), \\
I_{p}=C^{\prime}\left[P\left(1-\cos ^{2} \theta \cos ^{2} \gamma-\sin ^{2} \theta \sin ^{2} \gamma\right)+(1-P)\right] .
\end{gathered}
$$

Here, $\theta$ is the angle between the direction of the incident $\mathrm{x}$ rays and the sample surface, $\alpha$ is the angle between the sample surface normal and the vector pointing in the direction of a vectorlike orbital, $\gamma$ denotes the angle between the sample surface normal and the normal vector of a planelike orbital, $P$ is the degree of polarization, and $C$ and $C^{\prime}$ are normalization constants. ${ }^{9}$ Note that $\alpha$ and $\gamma$ are related to the corresponding tilt angles of the molecules through $90^{\circ}-\alpha$
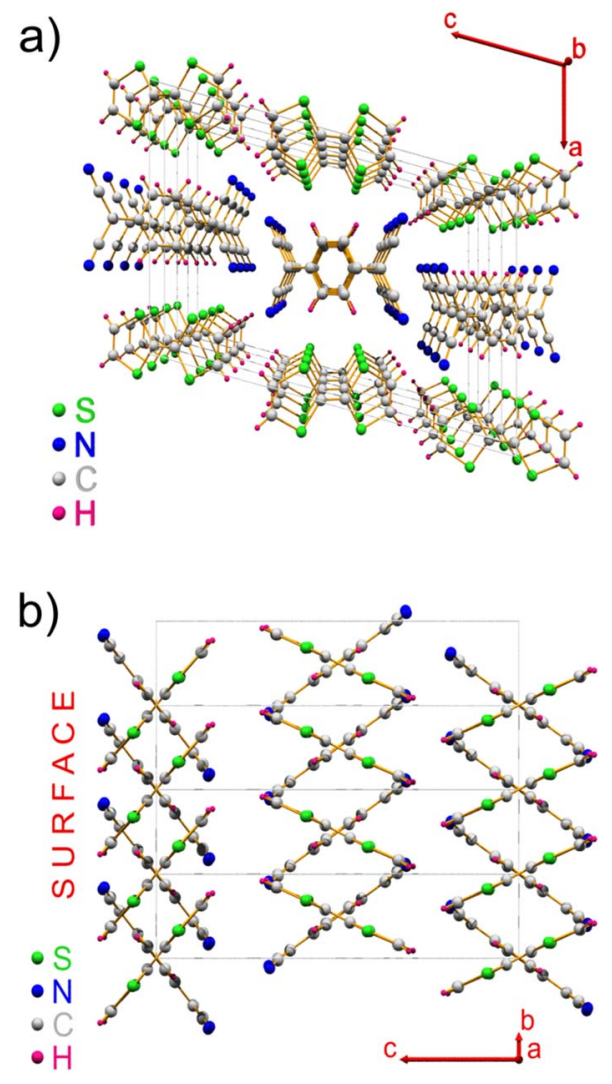

FIG. 1. (Color online) (a) Central projection view of the crystal structure of TTF-TCNQ along the $b$ axis. (b) Parallel projection view along $a$ with a hypothetical change in the molecule tilt angles at the surface. For details, see text.

and $90^{\circ}-\gamma$, respectively. The tilt angles can be determined by this method to an accuracy of about $\pm 10^{\circ}$.

In order to study the effect of surface relaxation on the hopping integral $t$, band calculations were performed within the standard density-functional theory (DFT) using the generalized gradient approximation (GGA). ${ }^{10}$ We employed the self-consistent full-potential linearized augmented plane wave method as implemented in the WIEN2K code. ${ }^{11}$

\section{STRUCTURAL AND ELECTRONIC PROPERTIES OF TETRATHIAFULVALENE- TETRACYANOQUINODIMETHANE}

Tetrathiafulvalene-tetracyanoquinodimethane (TTFTCNQ) crystallizes in a monoclinic structure (see Fig. 1), space group $P 2_{1} / c$, with lattice parameters $a=12.298 \AA, b$ $=3.819 \AA, c=18.468 \AA$, and $\beta=104.46^{\circ} .{ }^{12}$ It consists of stacks of either TTF or TCNQ molecules which run along the crystallographic $b$ axis. Quasi-one-dimensional bands are formed through overlapping $\pi$-type molecular orbitals which extend over the entire molecules. Maximum hopping probability along the stacks, and hence gain in kinetic energy, is accomplished by a tilt of the essentially planar and rigid molecules around the $a$ axis by $24.5^{\circ}$ and $34.0^{\circ}$ for the TTF and TCNQ molecules, respectively. The tilt angle is oppositely oriented between adjacent stacks. Metallicity arises 


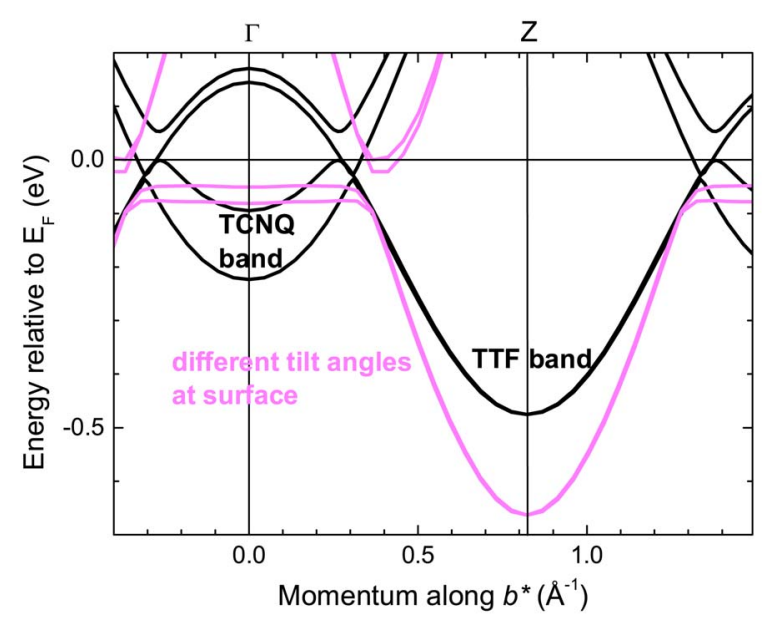

FIG. 2. (Color online) Bulk GGA band structure of TTF-TCNQ along the 1D direction for the real (black solid lines) and a hypothetical (pink or gray solid lines) band structure based on enlarged molecule tilt angles. For details, see text.

through charge transfer of about $0.6 e$ per TTF to each TCNQ molecule. A detailed description of the strongly anisotropic band structure as obtained by GGA calculations can be found elsewhere. ${ }^{4}$ Here, we focus on the TTF- and TCNQ-derived conduction bands just below the Fermi energy $E_{F}$ along $b$, i.e., along $\Gamma Z$ in the first Brillouin zone (black lines in Fig. 2). Around $\Gamma$, a TCNQ-derived band doublet (split by hybridization of the electronic states of the two stacks per unit cell) can be identified, while the corresponding almost degenerate TTF-derived doublet is found toward the Brillouin zone boundary. What is important in our context is the fact that the experimental ARPES dispersions indicate a band width larger by a factor of $\approx 2$ with respect to band theory. 3,4 This discrepancy might be reconciled by the assumption of a relaxed topmost molecular layer. At the surface, the Madelung potential and the polarization screening are different from the bulk, which may allow for different molecule tilt angles to minimize energy. Larger tilt angles lead to a smaller intermolecular distance $d$ and hence an increased transfer integral, which is counteracted by a reduced area overlap through a larger lateral offset $l$ of adjacent molecules in a stack. Assuming explicitly a $1 / d^{3}$ dependence ${ }^{13,14}$ of the transfer integral, this simple consideration leads to estimates for renormalized tilt angles as required to reproduce the experimental band widths, viz., $50^{\circ}$ and $48.3^{\circ}$ for TCNQ and TTF molecules, respectively. In order to explore this idea of a possible surface relaxation further, we have performed DFT calculations for a hypothetical bulk structure based on these tilt angles (see pink or gray solid lines in Fig. 2). While these indeed almost replicate the observed larger TTFderived conduction band width, the occupied part of the TCNQ band around $\Gamma$ comes out to have hardly any dispersion. Probably, this is due to the complex nodal structure of the lowest unoccupied molecular orbital (LUMO) of TCNQ ${ }^{5}$ perpendicular to the molecular plane, which was neglected in the above qualitative consideration but can modulate the otherwise slowly varying functional behavior of $|t|$ in an oscillating manner causing one or several minima. ${ }^{15}$ In any case, one can induce from the above that (i) a molecular surface relaxation with $d$ and $l$ values in a realistic parameter regime can indeed be responsible for the enhanced experimental band widths and (ii) that a sizable increase in the tilt angles by $\sim 20^{\circ}$ would be necessary. We add that it was briefly noted in the context of recent GGA calculations that a structural optimization for the outer molecular layer keeping the others fixed found changes in the molecule inclination angles though with minor consequences for the electronic dispersions along $b .^{16}$

\section{ANGULAR-DEPENDENT NEAR-EDGE X-RAY ABSORPTION FINE STRUCTURE SPECTRA}

The site and element sensitivity of NEXAFS enables the determination of the TTF and TCNQ surface tilt angles separately recording the $\mathrm{S} L$ and $\mathrm{N} K$ absorption edges, respectively. On the other hand, the angular dependence of the $\mathrm{C} K$ edge spectra contains information about the orientation of both molecules provided that the numerous resonances can be disentangled. In order to accurately extract the angular dependence of a certain resonance feature, the NEXAFS spectra have to be analyzed using a suitable fitting scheme. We followed the approach of Outka and Stöhr ${ }^{17}$ and fitted the spectra after a proper background subtraction with a minimal number of Gaussian- and Lorentzian-like peaks. Absorption into the free-electron continuum was accounted for by an error function step where appropriate, whose position and width reflect the binding energy and the relative chemical shift $^{18}$ due to the various inequivalent atoms in the structure. No "hidden" peaks were included in the fitting at a certain angle unless there was a definitive indication for them in the spectra at neighboring angles and a remnant still seen in the spectrum to be fitted. Ideally, during the fitting of a complete angle series, the parameters for all peaks (including the continuum step) are determined once and for all for a particular spectrum and then held fixed except for the ones describing the relative integral weight of each component. In practice, this cannot be sustained all over, especially for the $C K$ edge where nine crystallographically different $C$ atoms contribute and a unique identification of each resonance for all angles becomes difficult. Moreover, a small relaxation (within a few percent) of all parameters was permitted in order to achieve better fits. For peak assignment, we relied on the comprehensive NEXAFS study of Fraxedas et al. on highly textured TTF-TCNQ films. ${ }^{16}$ Note that throughout this paper $\sigma^{*}$ and $\pi^{*}$ resonances correspond to plane- and vectorlike molecular orbitals within the classification scheme of Ref. 9. Employing Eq. (2) to fit the angular intensity variation of a resonance excitation into a planelike orbital, we assumed a polarization degree $P \approx 1$.

In the left panel of Fig. 3, N $K$ absorption spectra are displayed for various light incidence angles $\theta$. On unbiased inspection, four resonances can be discerned which are numbered and denoted according to their symmetry. Their intensity variations with different light incidence angles are clearly seen. The N $K$ edge spectra represent the "clean" case, i.e., all spectra can be fitted with one single set of underlying components. An example of such a fit analysis 

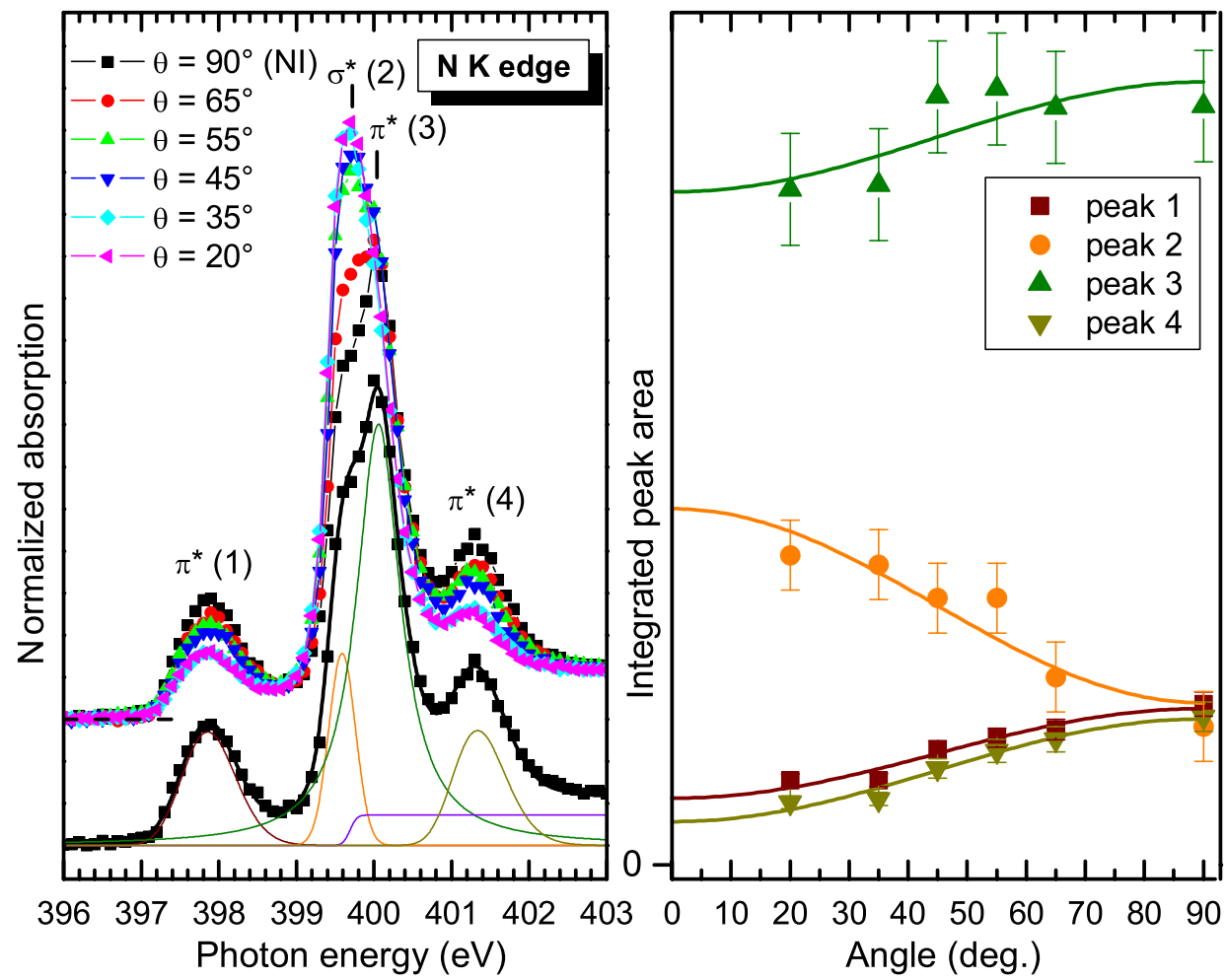

FIG. 3. (Color online) Left panel: $\mathrm{N} K$ edge NEXAFS spectra of TTF-TCNQ for various light incidence angles $\theta$. In the lower part, the normal incidence (NI) spectrum is reproduced together with the corresponding fit analysis from which the integral peak intensities are obtained. Right panel: Integrated peak areas for the peaks as in the fit of the left panel as a function of light incidence angle (scattered curves). The solid lines mark fits according to the model as described in the text.

with the total fit curve and its decomposition into the various constituents is depicted in the lower part of the panel for $\theta$ $=90^{\circ}$. The integral intensities of all four peaks are plotted in the right panel of Fig. 3 as a function of $\theta$ together with regression curves according to Eqs. (1) and (2). As tilt angles for the TCNQ molecules, one thus obtains $33.2^{\circ}\left(34.0^{\circ}\right.$, $\left.42.8^{\circ}, 28.6^{\circ}\right)$ for peak $1(2,3,4)$. These values are in fair agreement with the number $34.0^{\circ}$ from the bulk. The somewhat higher value derived from the intensity variation of peak 3 probably reflects the insufficient approximation of the underlying spectral weight by just one peak as can be seen from its relative broad Lorentzian shape. Actually, most of the resonances which are denoted here comprise more than one molecular orbital with the same symmetries. ${ }^{16}$ However, this shortcoming has to be accepted following the directive to use a minimal set of peaks, for all of which clear indication is to be seen in at least one spectrum.

Figure 4 shows $S L$ edge NEXAFS spectra which are specific for TTF. As before, distinct intensity variations as a function of $\theta$ are observed for at least four resonances which again are marked according to their symmetry in the plot. A detailed fit analysis is hampered here for two reasons. First, the signal-to-noise ratio is much worse in the Auger yield detection mode as compared to the $\mathrm{N} K$ edge, which, however, cannot be easily compensated by longer measuring times due to the comparatively rapid radiation induced sample degradation. Second, the overall spectral shape is much broader and the individual resonance peaks are much less well separated than in the case of the $\mathrm{N} K$ edge spectra except for the $\sigma^{*}$ resonance at $164.8 \mathrm{eV}$. Therefore, we only fitted this single peak for the evaluation of the TTF tilt angle. From the fit of the angle-dependent integral intensity which is displayed in the right panel of Fig. 4 , a value of $15.7^{\circ}$ is obtained again in reasonable agreement with the bulk value of $24.5^{\circ}$ and at variance with the expectation in the case of a sizable surface relaxation.

The results obtained so far for the TTF and TCNQ tilt angles might be corroborated from an analysis of the $\mathrm{C} K$ spectra if one succeeds to trace the intrinsic angular dependence of a resonance located either on the TTF or TCNQ molecules. In Fig. 5, C $K$ spectra taken at different light incidence angles are plotted. By comparison of $\mathrm{C} K$ edge spectra for TTF, TCNQ, and TTF-TCNQ shown in Ref. 16, we can grossly label few structures in our data according to their symmetry and origin from either TTF or TCNQ. From the fit analysis - a representative regression curve is plotted in the lower part of the left panel in Fig. 5-it turns out that a unique tracking is only possible for the most pronounced resonance structure at $285.6 \mathrm{eV}$, which is identified as a $\pi^{*}$ resonance on TTF. In the right panel, the angular variation of its integral intensity is shown together with the corresponding fit curve giving a tilt angle of $27.8^{\circ}$. This again reflects the bulk value of $24.5^{\circ}$ in agreement with the analysis of the S $L$ edge spectra instead of a strongly increased tilt for a relaxed surface. 

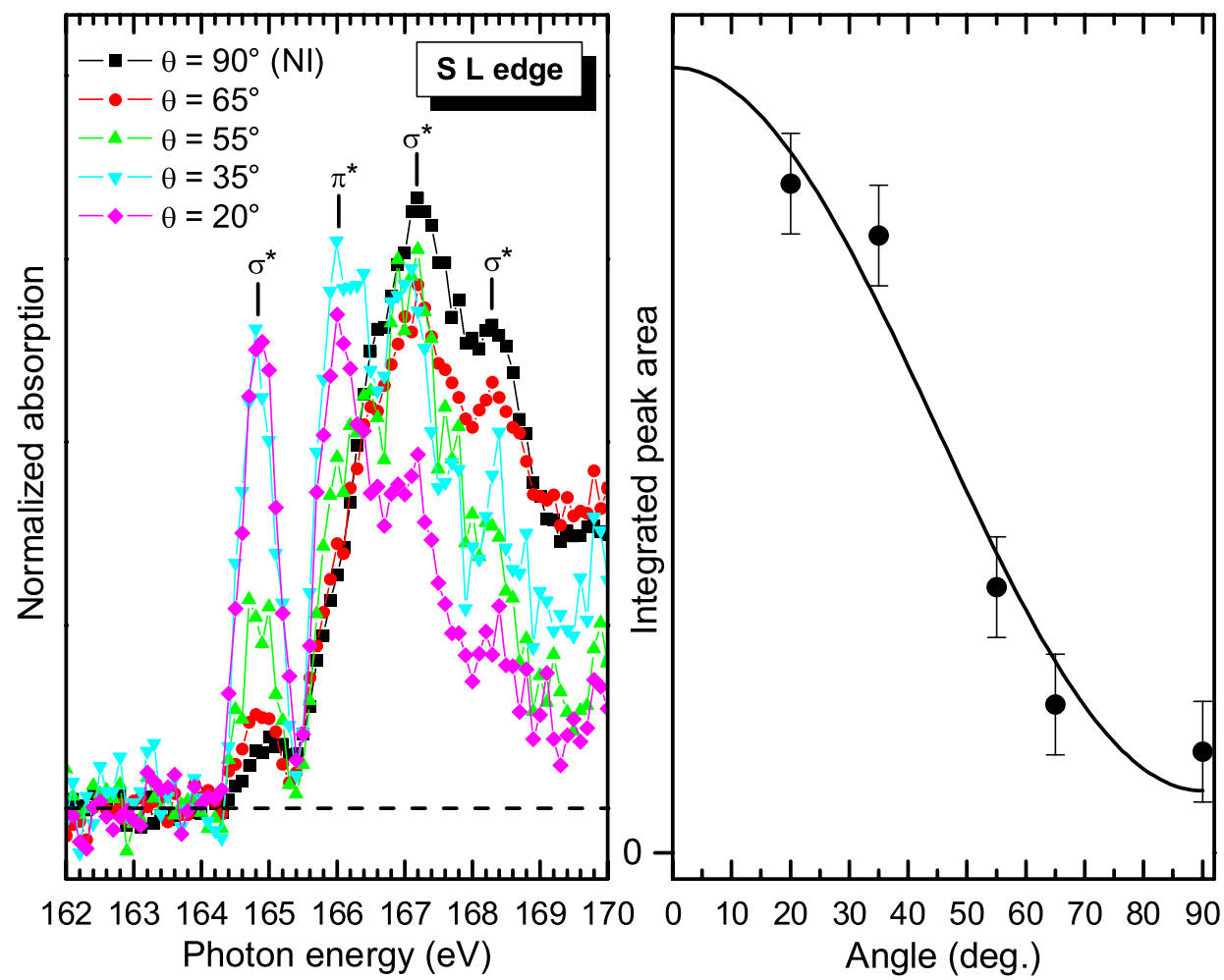

FIG. 4. (Color online) Left panel: S $L$ edge NEXAFS spectra of TTF-TCNQ for various light incidence angles $\theta$. Right panel: Integrated area for the pronounced $\sigma^{*}$ resonance peak at $164.8 \mathrm{eV}$ (dotted curve). The solid line depicts a fit according to the model as described in the text.

\section{DISCUSSION}

In the search of experimental realizations of generically 1D systems, many claims have been made on the observation of certain isolated aspects such as spin-charge separation or the nonuniversal power-law suppression of spectral weight at the chemical potential. However, it is fair to say that a comprehensive theoretical and experimental picture where a sufficient number of pieces are put together thus exhibiting clear 1D phenomenology has not yet been achieved for any of these systems. Probably for TTF-TCNQ, such an understanding is farthest developed. There, not only the electronic ARPES dispersions of the TCNQ related spectral weight could be quantitatively fitted by the spinon and holon branches of the 1D HM using the Bethe ansatz solution ${ }^{4}$ and the pseudofermion dynamical theory ${ }^{19}$ but also the overall spectral weight distribution could be reproduced by densitymatrix renormalization-group calculations. ${ }^{2}$ The underlying parameter set in both cases, viz., $U=1.96 \mathrm{eV}$ and $t=0.4 \mathrm{eV}$, however, assumes a hopping integral $t$ which is enhanced by a factor of $\approx 2$ with respect to the DFT calculations. To justify this assumption, it was argued from an experimental point of view that the topmost surface layer of TTF-TCNQ might be relaxed. ${ }^{3,4}$ On the other hand, further theoretical work has shown that the also observed transfer of spectral weight at $k_{F}$ over the entire conduction band width with increasing temperatures cannot be reconciled within this parameter set. ${ }^{6,20}$ The effective magnetic exchange $J_{\text {eff }}$ which defines a characteristic temperature for the onset of such an spectral-weight transfer would come out much too large within the 1D HM. This questions the assumption of a surface relaxation and hence an enhanced value of $t$ to explain the experimental data. Results by exact diagonalization ${ }^{6}$ for $T=0$ found that the inclusion of the nearest-neighbor Coulomb repulsion $V$ in the extended HM offers a possible loop hole since it effectively increases the band widths of the spinon and holon excitations to match the experimental dispersions while, at the same time, allowing for a smaller $t$ and hence a smaller $J_{\text {eff }}$ in better agreement with experiment. Indeed, Cano-Cortés et al. ${ }^{5}$ recently have reported on density-functional results which find that the long-ranged Coulomb repulsion even to the third nearest neighboring molecule within a stack is still about $20 \%$ of $U$. However, these results are based on the bulk crystal structure of TTFTCNQ. In this context, the NEXAFS work presented here provides the missing link which is needed to reconcile ARPES dispersions and $T$ dependence with theory, viz., HM and DFT calculations. Our results indicate that no surface relaxation, i.e., no extra molecule tilt, occurs for either the TTF or the TCNQ stacks, leaving the hopping integral $t$ at the surface essentially unaltered with respect to the bulk. These findings validate the work by Cano-Cortés et al., which, in turn, corroborates the theoretical conclusion concerning the temperature-dependent redistribution of spectral weight above $J_{\text {eff }}$. It should not be concealed here that a theoretical description of the complete ARPES data is not yet accomplished. In particular, the TTF derived part of the data calls for a consistent treatment on the same footing. This 

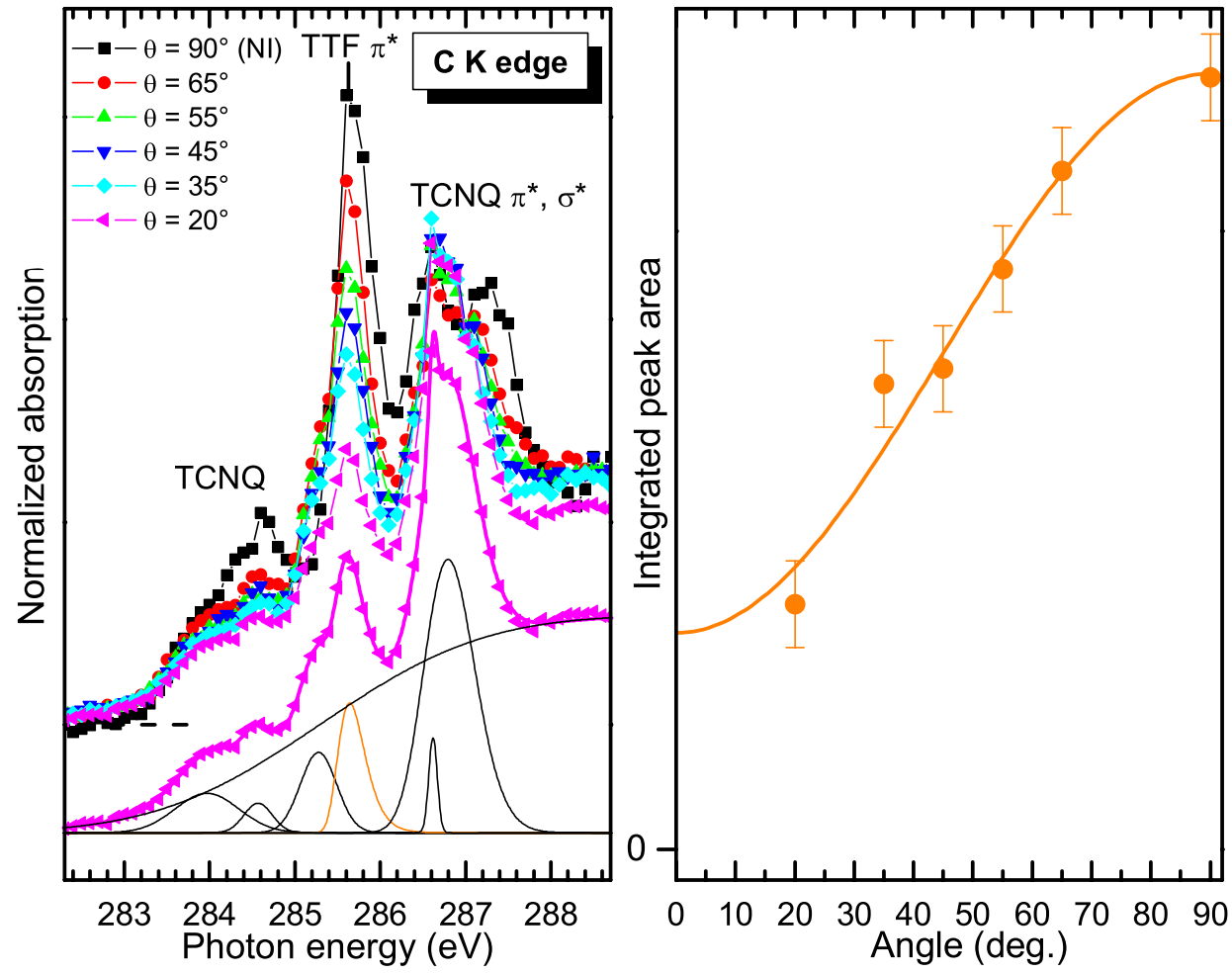

FIG. 5. (Color online) Left panel: C $K$ edge NEXAFS spectra of TTF-TCNQ for various light incidence angles $\theta$. In the lower part, the spectrum for $\theta=20^{\circ}$ is reproduced together with the corresponding fit analysis from which the integral peak intensities were obtained. Right panel: Integrated peak area for the peak marked in orange or gray in the left panel as a function of light incidence angle (dotted curve). The solid line depicts a fit according to the model as described in the text.

should be able to explain, e.g., the seeming absence of the spinon-holon splitting, although the electronic states of the TTF stacks should be more strongly correlated than those of the TCNQ stacks.

\section{CONCLUSIONS}

From the analysis of the angular dependence of the $\mathrm{N} K$, $\mathrm{S} L$, and C $K$ NEXAFS spectra, we arrive at a consistent picture where, under the restrictions imposed by experiment, the topmost molecular layer of TTF-TCNQ single crystals appears to be structurally unaltered with respect to the bulk. This result lends additional significance to the approach to explain the deviations between experimental and DFT band dispersions purely electronically. A refinement of the singleband 1D Hubbard model by inclusion of a next-neighbor Coulomb repulsion $V$ as proposed to explain the temperature dependence of the photoemission data thus seems plausible from an experimental point of view.

\section{ACKNOWLEDGMENTS}

We are grateful to M. Sperling for technical assistance during the experiments at BESSY and thank A. Schöll and E. Koch for fruitful discussions. The experimental end station was funded under MUSTANG BMBF $05 \mathrm{KS} 4 \mathrm{OC} 1 / 3$. *michael.sing@physik.uni-wuerzburg.de

${ }^{1}$ J. Voit, Rep. Prog. Phys. 58, 977 (1995).

${ }^{2}$ H. Benthien, F. Gebhard, and E. Jeckelmann, Phys. Rev. Lett. 92, 256401 (2004).

${ }^{3}$ R. Claessen, M. Sing, U. Schwingenschlögl, P. Blaha, M. Dressel, and C. S. Jacobsen, Phys. Rev. Lett. 88, 096402 (2002).

${ }^{4}$ M. Sing, U. Schwingenschlögl, R. Claessen, P. Blaha, J. M. P. Carmelo, L. M. Martelo, P. D. Sacramento, M. Dressel, and C. S. Jacobsen, Phys. Rev. B 68, 125111 (2003).

${ }^{5}$ L. Cano-Cortés, A. Dolfen, J. Merino, J. Behler, B. Delley, K. Reuter, and E. Koch, Eur. Phys. J. B 56, 173 (2007).
${ }^{6}$ N. Bulut, H. Matsueda, T. Tohyama, and S. Maekawa, Phys. Rev. B 74, 113106 (2006).

${ }^{7}$ J. Stöhr, NEXAFS Spectroscopy, Springer Series in Surface Sciences Vol. 25 (Springer, Berlin, 1996).

${ }^{8}$ For a definition of vector- and planelike orbitals, see Ref. 9.

${ }^{9}$ J. Stöhr and D. A. Outka, Phys. Rev. B 36, 7891 (1987).

${ }^{10}$ J. P. Perdew, K. Burke, and M. Ernzerhof, Phys. Rev. Lett. 77, 3865 (1996).

${ }^{11}$ P. Blaha, K. Schwarz, G. K. H. Madsen, D. Kvasnicka, and J. Luitz, Wien2k; An Augmented Plane Wave Plus Local Orbitals Program for Calculating Crystal Properties (Vienna University 
of Technology, Vienna, Austria, 2001).

${ }^{12}$ T. J. Kirstenmacher, T. E. Phillips, and D. O. Cowan, Acta Crystallogr., Sect. B: Struct. Crystallogr. Cryst. Chem. B30, 763 (1974).

${ }^{13}$ O. K. Andersen, H. L. Skriver, H. Nohl, and B. Johansson, Pure Appl. Chem. 52, 93 (1979).

${ }^{14}$ Depending on the theoretical approach, one finds different scaling behaviors for the transfer integral $t$ in the literature ranging from a power law decay as $1 / d^{2}$ to an exponential decay. However, these discrepancies do not affect our qualitative arguments.

${ }^{15}$ P. M. Kazmaier and R. Hoffmann, J. Am. Chem. Soc. 116, 9684
(1994).

${ }^{16}$ J. Fraxedas, Y. J. Lee, I. Jiménez, R. Gago, R. M. Nieminen, P. Oredejón, and E. Canadell, Phys. Rev. B 68, 195115 (2003). ${ }^{17}$ D. A. Outka and J. Stöhr, J. Chem. Phys. 88, 3539 (1988).

${ }^{18}$ M. Sing, U. Schwingenschlögl, R. Claessen, M. Dressel, and C. S. Jacobsen, Phys. Rev. B 67, 125402 (2003).

${ }^{19}$ J. M. P. Carmelo, K. Penc, L. M. Martelo, P. D. Sacramento, J. M. B. L. D. Santos, R. Claessen, M. Sing, and U. Schwingenschlögl, Europhys. Lett. 67, 233 (2004).

${ }^{20}$ A. Abendschein and F. F. Assaad, Phys. Rev. B 73, 165119 (2006). 